\title{
Hospital information system integration based on cloud computing
}

\author{
YAO Qin, WANG Yu, LI Jing-song* \\ Healthcare Informatics Engineering Research Center, \\ Key Laboratory for Biomedical Engineering of Ministry of Education, \\ Zhejiang University, Hangzhou, 310027, China; \\ *Towhom correspondence should be addressed; e-mail: 1js@zju.edu.cn
}

\begin{abstract}
With the development of hospital information construction, medical information systems are becoming more and more diversified and complicated in hospitals. The traditional architecture in which a single application is supported by single or multiple servers will largely increase the number of servers, the total cost of ownership, and the difficulty of centralized management. By analyzing the core technology and service mode of cloud computing, this paper constructs a health information integration model based on cloud computing. On the basis of this model, we develop a health information integration platform in private cloud with electronic medical record (EMR) as the core.
\end{abstract}

Keywords-Cloud computing; virtualization ; integration platform

\section{INTRODUCTION}

The development of medical information technology brings great opportunities and challenges. In order to make the hospital run properly, the engineers should solve the problems of deploying, integrating and managing the medical information systems and other related systems, which is involved in plenty of complicated hardwares and softwares.

The different medical information systems usually require different system architectures to support, so no one is always the best choice. Cloud computing is available ondemand and provides flexible and scalable computing resources by integrating the IT infrastructure [1]. The developers begin to move medical information systems to the Cloud [2, 3]. Using server virtualization, we can virtualize all kinds of visual machines (VMs) needed for different kinds of medical information systems, realizing the rapid deployment. Using desktop virtualization, we can integrate medical applications into desktops and deliver them to the users. On the basis of integration of hardware resources by cloud computing, we simultaneously have to establish an integration platform which is up to Healthcare Level 7 (HL7) Standard [4] on the cloud to realize the information interoperability between different systems.

\section{THE ARCHITECTURE}

The Architecture of integration platform in private cloud has three core parts: the distributed storage, the server cluster and virtualization, as shown in Figure 1. The distributed storage is established and managed by Hadoop Distributed File System (HDFS) [5]. All of the medical information systems and the integration platform are implemented in the VMs that virtualized by VMware vSphere [6] tools. The developers and maintenance engineers manage the entire infrastructure though the management server. The medical information systems users obtain services though desktop virtualization, which seems just like before.

Hadoop provides HDFS that stores data on the compute nodes, providing very high aggregate bandwidth across the cluster [7]. In addition, it works both on the physical computer and VMs [8].

Server virtualization provides all kinds of operation systems (OS), such as LINUX, Windows, Macintosh, which are needed by the different systems during installation. With the aid of the virtualization, multiple VMs share a single physical computer in order to improve the utilization rate of resources. Server Virtualization separates the infrastructure with the OS environment that the medical information systems deployed, so it can reduce the difficulty and the time of the system deployment.

Desktop virtualization is based on the VMs that provided by server virtualization. Desktop virtualization offers a way operating one desktop in multi-user mode without interference, it can simplify the deployment process of medical system, reduce the late maintenance difficulty and cost.

The Integration Platform is designed and build on VMs that provided by server virtualization. It establishes medical data communication between all kinds of medical information systems based on HL7 v2.4 standard, combined with File, FTP, SQL, TCP, et al, and other technical means to establish all kinds of adapters to meet the diversity of the medical system interfaces. Implemented with Integrating the Healthcare Enterprise (IHE) technical framework in both internal and external integration platform to regulate the workflow patterns of medical data integration, ensure the consistency of data between various kinds of systems.

\section{BUILDING THE PRIVATE CLOUD}

\section{A. Instrallation Environment}




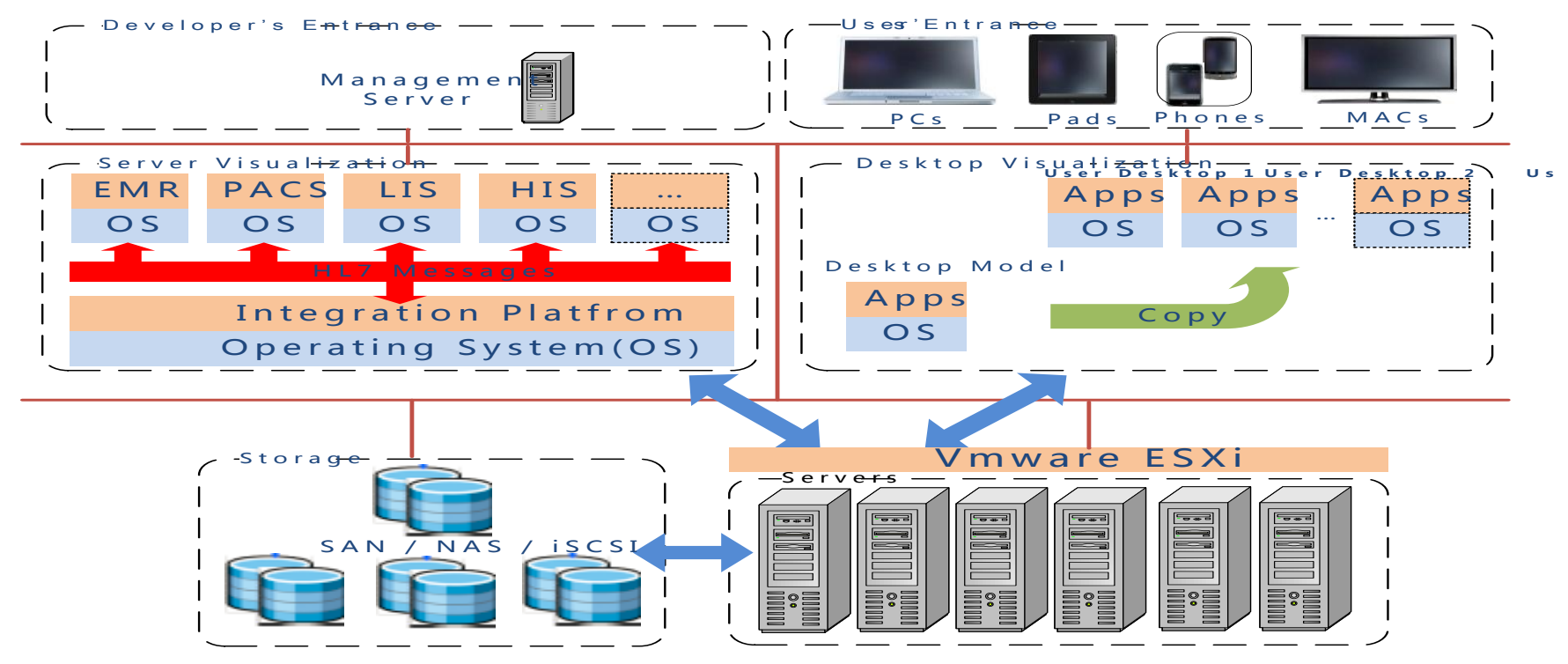

Figure 1. The Architecture of integration platform in private cloud

This paper mainly adopts the VMware vSphere 5.1 and VMware Horizon View 5.2 to build the private cloud. Before installing, we should prepare the vSphere environment.

The whole experiment environment required for the virtual machine, the machine list, include the machine name, IP address and operating system, etc. As shown in TABLE I.

TABLE I. INSTALLATION ENVIRONMENT

\begin{tabular}{|c|c|c|c|}
\hline Role & OS & Name & IP \\
\hline $\begin{array}{c}\text { Domain } \\
\text { Controller }\end{array}$ & Win Server 2008 R2 x64 & bme.com & $\begin{array}{c}10.13 .22 .1 \\
69\end{array}$ \\
\hline $\begin{array}{c}\text { vCenter } \\
\text { View }\end{array}$ & Win Server 2008 R2 x64 & $\begin{array}{c}\text { vCenter.b } \\
\text { me.com }\end{array}$ & $\begin{array}{c}10.13 .22 .1 \\
61\end{array}$ \\
\hline ESXi 1 & ESXi 5.1 & $\begin{array}{c}\text { esxi01.bm } \\
\text { e.com }\end{array}$ & $\begin{array}{c}10.13 .22 .1 \\
62\end{array}$ \\
\hline ESXi 2 & ESXi 5.1 & $\begin{array}{c}\text { esxi02.bm } \\
\text { e.com }\end{array}$ & $\begin{array}{c}10.13 .22 .1 \\
63\end{array}$ \\
\hline $\begin{array}{c}\text { Connection } \\
\text { Server }\end{array}$ & Win Server 2008 R2 x64 & - & $\begin{array}{c}10.13 .22 .1 \\
51\end{array}$ \\
\hline $\begin{array}{c}\text { Security } \\
\text { Server }\end{array}$ & Win Server 2008 R2 x64 & - & $\begin{array}{c}10.13 .22 .1 \\
71\end{array}$ \\
\hline $\begin{array}{c}\text { VMware } \\
\text { View Client }\end{array}$ & Windows 7 & $\begin{array}{c}\text { view.bme. } \\
\text { com }\end{array}$ & $\begin{array}{c}10.12 .43 .9 \\
1\end{array}$ \\
\hline
\end{tabular}

\section{B. Implementation of Private Cloud}

In order to build the private cloud, we should install all the roles that shown in TABLE I. Then, we use server virtualization to create all the VMs needed for the medical information systems and the desktop users. In this paper, we use ESXi 1 to build server pool, and ESXi 2 to build desktop pool. All the servers of medical information systems are running on the VMs virtualized on ESXi 1. All the clients or applications of the systems are running on the VMs virtualized on ESXi 2 (Figure 2).

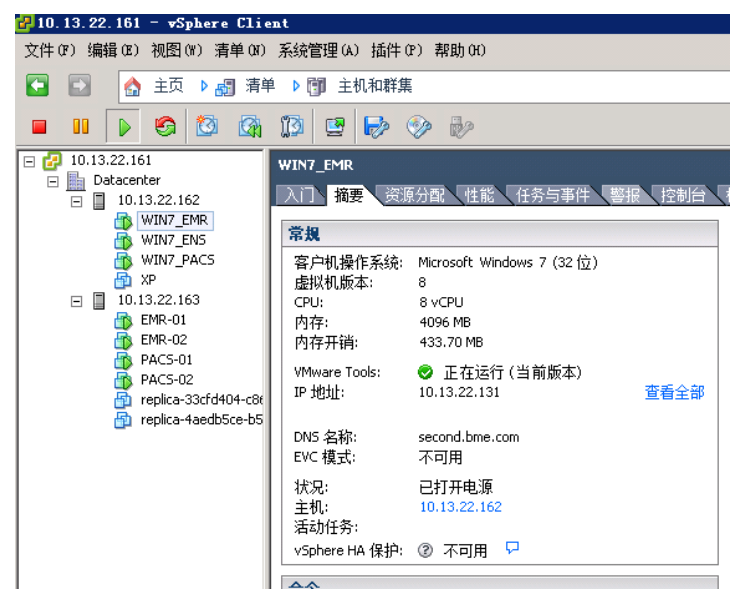

Figure 2. Implementation of Private Cloud

\section{DESIGN OF THE PLATFORM}

Medical information should be exchanged and shared through heterogeneous medical information systems in hospital. Integration platform needs stable environment to exchange data between the medical information systems. It has to deal with not only very complicated hardware environment, but also the various interfaces connected with different medical information systems. In non-cloud environment, development and implement of the platform is very difficult.

In this paper, we design an integration mode that combined with VMs, using Ensemble [9]. Ensemble gathers data server, integration server, application server and portal development software in one, can achieve the coordination of heterogeneous data and business process reengineering, quickly build new business productions, and reduce the complexity of integrating the medical information integration. 


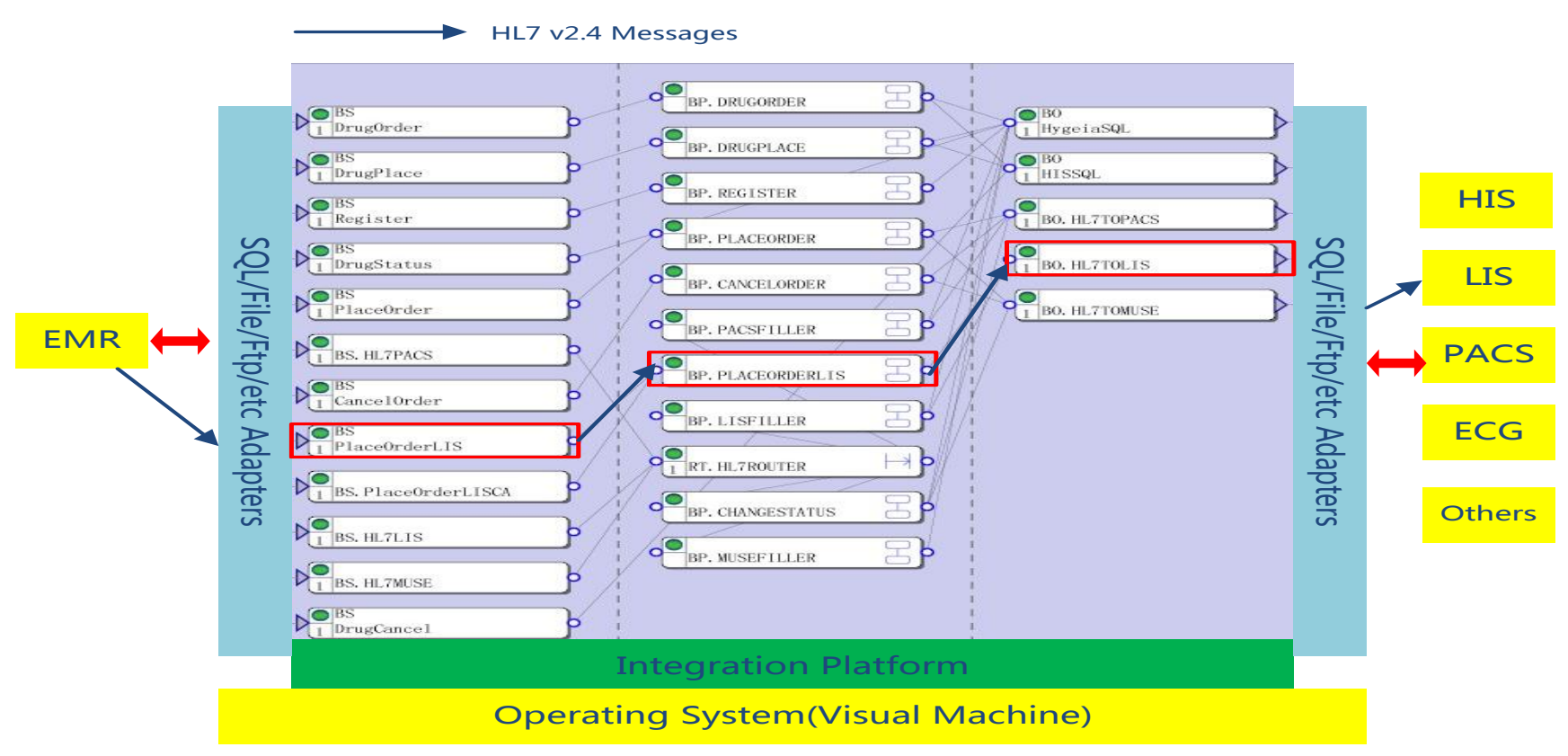

Figure 3. Design of Integration Platform On VM

All the components of the integration platform are virtualized on the cloud, as shown in Figure 3.

HL7 standard as an important criterion of the application and implementation of medical information system, can achieve the standards of the data transmission by using message, standardize business processes, restrain the medical practices, ensure the quality of medical care, make the authenticity and validity of information.

The function of integration platform is integrating the medical information and standardizing data with HL7, the framework as Figure 3. It can realize to acquire, organize and encapsulate the data which from EMR, hospital information system (HIS), picture archiving and communication system (PACS), laboratory information system (LIS), etc. Due to different systems may have different interfaces, we set up all kinds of inbound or outbound adapters to connect and communicate with the different medical information systems though the integration platform. At the same time, though the data standardized interface inside the integration platform, we use HL7 standard to do the standardized processing of medical information and unified management. The integration platform can also transform the HL7 messages to other non-standardized messages that used by some specific systems.

Every single process of health affairs, such as Patient Registration, Placing PACS Order, is independent in the integration platform. In consequence of such design, the integration platform can be implemented on distributed systems, which can reduce the consumption of the integration platform and increase the capability. With the help of VMs, distributed implementation of integration platform components becomes easier and will not cause more physical resource cost.

Comparing the VMs used by integration platform with others used by medical information systems, they require more network capacity than storage space. In private cloud, server virtualization can distribute these resources appropriately.

\section{RESULT OF INTEGRATION PLATFORM}

After all the medical information systems and the components of the integration platform have been implemented on their VMs. The integration platform connect $\mathrm{s}$ these systems in a loosely way, using HL7 v2.4 Messages, as shown in Figure 4.

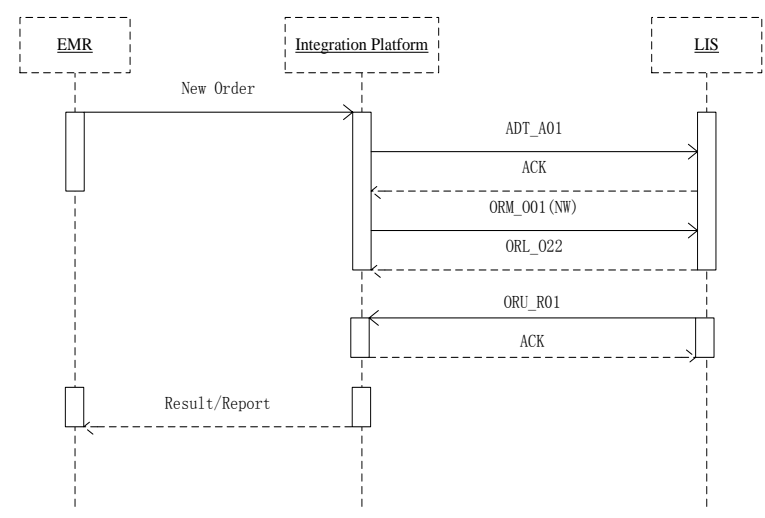

Figure 4. A Simplified IHE Model of Integration with EMR and LIS

A doctor uses his own devices that can access the desktop virtualized in the private cloud, which also implemented with EMR client, to issue a LIS order; The integration platform receives an EMR-message though its inbound adapter, transforms it to HL7 Messages (ADT_A01, ORM_O01), and delivers these messages to LIS by FTP 
Adapter. After the patient was treated by the LIS doctors who also obtain desktop service implemented with LIS client from the private cloud, the LIS result will be sent back to the doctor issued the order though another process in the integration platform, which can be set up on the same or another different VM.

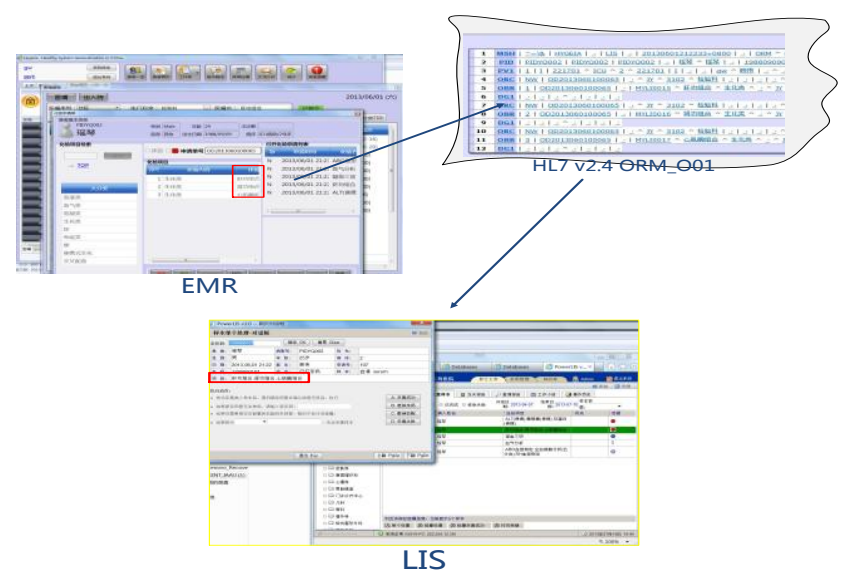

Figure 5. Information Exchange between EMR and LIS

\section{CONCLUSION AND DISCUSSION}

The architecture of integration platform in private cloud is aim to solve the problems and difficulties that the developers and maintenance engineers meet in implementing and integrating the medical information systems in hospitals.

The private cloud provides the virtualized environments for the systems rapidly by VMs. The integration platform which can also works on a single or multiple VMs helps the medical information systems to interoperate each other in a stable and loosely way. Meanwhile, desktop virtualization in the cloud can help us to deliver the applications to the end user easily. The users can obtain these service buy any pieces of their devices, like Phones, Pads, PCs, MACs and so on, whenever and wherever as soon as network is available.

The systems, applications, and the integration platform are once set up in the private cloud appropriately. They are encapsulated into the VMs. Because the VMs are stored in the physical computer in form of files, backup and reinstallation of these medical information system becomes very simple. This design could actually reduce the system deployment cost and time.

Moreover, there are still a lot of systems for hospital are being developed or waiting for being implemented. In traditional computing architectures, firstly, we have to buy servers and other equipment required by the new system; secondly, we have to redesign and rebuild the integration platform to adapt to new business requirements. These steps cost a lot and waste a lot. In this architecture, we can provide the entire environment for the new system immediately with VMs. The integration platform also do not need to be rebuild, because we can develop and add these new integration processes with VMs directly without influencing the existing processes.

This architecture provides a flexible, resilient approach to deploy, manage, maintain and integrate the whole hospital information infrastructure.

\section{ACKNOWLEDGMENT}

This work was supported by the National Natural Science Foundation (Grant No. 61173127) and National High-tech R\&D Program (No. 2013AA041201) and Zhejiang University Top Disciplinary Partnership Program (Grant No. $188170 * 193251101)$.

\section{REFERENCES}

[1] M. Armbrust, A. Fox, R. Griffith, A. D. Joseph, R. Katz, A Konwinski, M. Zaharia, A view of cloud computing. Communications of the ACM, 2010, 53(4), 50-58.

[2] G. C. Kagadis, C. Kloukinas, K. Moore, J. Philbin, P. Papadimitroulas, C. Alexakos, W. R. Hendee, Cloud computing in medical imaging. Medical physics, 2013, 40, 070901.

[3] L. A. Silva, C. Costa, J. L. Oliveira, A PACS archive architecture supported on cloud services. International journal of computer assisted radiology and surgery 7.3 (2012): 349-358.

[4] HL7 Standard. http://www.hl7.org/.

[5] Apache Hadoop. http://hadoop.apache.org/.

[6] VMware. http://www.vmware.com/.

[7] K. Shavachko, H. Kuang, S. Radia, R. Chansler, The hadoop distributed file system. Mass Storage Systems and Technologies (MSST), 2010 IEEE 26th Symposium on. IEEE, 2010.

[8] I. Masakuni, J. Han, H. Makino, Design and performance evaluation for Hadoop clusters on virtualized environment. Information Networking (ICOIN), 2013 International Conference on. IEEE, 2013.

[9] Ensemble. http://www.intersystems.com/ensemble/index.html. 